Himalayan J. Soc. Sci. \& Humanities ISSN: 0975-9891

Vol. 14, (2019) 33-39

DOI: https://doi.org/10.51220/hjssh.v14il.5

\title{
Life Portrayed In Regional Novels Of Hindi
}

\section{Rakhi Upadhyay}

Department of Hindi, DAV PG College, Dehradun, Uttarakhand.

*Corresponding Author Email: drrakhi.418@gmail.com

Received: 8.6.2019; Revised: 21.8.2019; Accepted: 2.10.2019

(C) Society for Himalayan Action Research and Development

\begin{abstract}
The element of regionalism in Hindi novels is very old. Novels that have an overall depiction of the life of a particular state are called regional novels. Regional novels are often called character-oriented, while in these novels, the focus of the author is not so much on the characters as it is about presenting the culture of that region. That is why some people call these a novel without a hero, and consider the region as important as hero. In the present communication a detailed description of public life depicted in these regional Hindi novels.
\end{abstract}

Keywords: Novel, Regionalism, Rural, Character Oriented, Folk culture, Language

\section{हिन्दी के आँचलिक उपन्यासों में चित्रित जन-जीवन राखी उपाध्याय}

हिन्दी विभाग, डी.ए.वी. (पी.जी.) कॉलेज, देहरादून, उत्तराखण्ड

*Corresponding Author Email: drrakhi.418@gmail.com

Received: 8.6.2019; Revised: 21.8.2019; Accepted: 2.10.2019

(C) Society for Himalayan Action Research and Development

सारांश

हिन्दी उपन्यासों में आँचलिकता का तत्त्व पर्याप्त पुराना है। जिन उपन्यासों में किसी विशिष्ट प्रदेश के जन-जीवन का समग्र बिम्बात्मक चित्रण हो, उन्हें आँचलिक उपन्यास कहा जाता है। आँचलिक उपन्यासों को प्रायः चरित्र प्रधान कहा जाता है, जबकि वस्तुतः इन उपन्यासों में लेखक का ध्यान पात्रों पर इतना नहीं होता जितना अंचल की संस्कृति को प्रस्तुत करने पर होता है। इसीलिए कुछ लोग उसे नायक-विहीन उपन्यास कहते हैं और अधिक से अधिक अंचल को ही उसका नायक मानते हैं। प्रस्तुत शोध पत्र में हिन्दी के आँचलिक उपन्यासों में चित्रित जन-जीवन का विवेचनात्मक प्रस्तुतीकरण किया गया है।

कुँजी शब्द - उपन्यास, आँचलिकता, ग्रामीण अंचल, चरित्र-प्रधानता, लोक संस्कृति, भाषा 
इस शताब्दी के पाँचवें-छठे दशकों में हिन्दी उपन्यास का एक नया रूप हमारे सामने आता है जिसे 'आँचलिक उपन्यास' कहा गया है। यद्यपि हिन्दी उपन्यासों में आँचलिकता का तत्त्व पर्याप्त पुराना है- प्रेमचन्द, वृंदावनलाल वर्मा, नागार्जुन आदि की रचनाओं में वह पहले ही मिलता है, पर उपन्यास को आँचलिक कहने तथा उसकी महत्ता पर लेखकों और आलोचकों का ध्यान आकृष्ट करने का श्रेय फणीश्वर नाथ रेणु और उनके 'मैला आँचल' नामक उपन्यास को है। सन् 1954 में प्रकाशित इस उपन्यास की भूमिका में लेखक ने लिखा था-

"वह है मैला आँचल एक आँचलिक उपन्यास कथानक पूर्णिमा। .... मैंने इसके एक हिस्से के एक ही गाँव को पिछड़े गाँवों का प्रतीक मानकर इस उपन्यास का कथा-क्षेत्र बनाया है।"

अंचल का अर्थ है जनपद या क्षेत्र। जिन उपन्यासों में किसी विशिष्ट प्रदेश के जन-जीवन का समग्र बिम्बात्मक चित्रण हो, उन्हें आँचलिक उपन्यास कहा जाता है। पाश्चात्य देशों में ऐसे उपन्यास तो लिखे गये जिनमें प्रदेश विशेष की भौगोलिक पृष्ठभूमि पर वहाँ की कतिपय विशेषताओं का दिगदर्शन किया गया हो, पर उन्हें आँचलिक नहीं कहा गया। ऐसे उपन्यासों की समीक्षा करते हुए आलोचकों ने प्रादेशिक रंग शब्द का प्रयोग किया था। कारण यह था कि इन उपन्यासों में प्रादेशिक तो थी पर लेखक का ध्यान प्रदेश विशेष से हटकर अन्यत्र भी गया था और उसने क्षेत्र विशेष की संस्कृति, सामाजिक, धार्मिक, राजनीतिक, आर्थिक स्थिति पर ही अपना ध्यान केन्द्रित नहीं किया था। उनके लैंस का फोकस केवल उसी बिन्दु पर नहीं पड़ा था। यही बात रेणु के 'मैला आँचल' से पहले प्रकाशित होने वाले शिव-पूजन सहाय के 'देहाती दुनिया' 2 तथा प्रेमचन्द-वृन्दावन लाल के उपन्यासों के सम्बन्ध में भी कही जा सकती है। अतः हिंदी में आँचलिक उपन्यास विशुद्ध भारतीय विधा है, उसे प्रेरणा भले ही पश्चिम से मिली हो, पर उसका स्वरूप और आकार भारतीय ही है।

आँचलिक उपन्यासों को प्रायः चरित्र प्रधान कहा जाता है, जबकि वस्तुतः इन उपन्यासों में लेखक का ध्यान पात्रों पर इतना नहीं होता जितना अंचल की संस्कृति को प्रस्तुत करने पर होता है। इसीलिए कुछ लोग उसे नायक-विहीन उपन्यास कहते हैं और अधिक से अधिक अंचल को ही उसका नायक मानते हैं। इस दृष्टि से आँचलिक उपन्यास चारित्रिक उपन्यास की परिधि में नहीं बाँधे जा सकतें इसी प्रकार न उन्हें सामाजिक ही कहा जा सकता है और न ऐतिहासिक ही। यह ठीक है कि उनमें सामाजिक समस्याओं का चित्रण होता है, पर उसके प्रणयन की प्रेरणा और प्रक्रिया सामाजिक उपन्यासों की सृजन-प्रक्रिया तथा प्रणयन-प्रेरणा से सर्वथा भिन्न होती है। सामाजिक उपन्यास में लेखक की दृष्टि सामाजिक समस्याओं के विशद चित्रण और विवेचन पर रहती है, जबकि आँचलिक उपन्यासकार सामाजिक समस्याओं का चित्रण अंचल विशेष को उभार कर प्रस्तुत करने के लिए और उसी की सीमाओं में ही करता है। प्रथम में सामाजिक समस्याएँ प्रधान होती है, दूसरे में गोण, सहायक उपकरण मात्र। उन्हें ऐतिहासिक उपन्यास कहना भी ठीक नहीं है। ऐतिहासिक उपन्यासों में काल-विशेष के जन-जीवन का चित्रण तो किया जाता है, पर वह पुस्तकीय ज्ञान के अनुमान पर आधारित होता है, स्वानुभव पर नहीं और आंचलिक उपन्यास के लिए स्वानुभव प्रत्यक्ष-दर्शन आवश्यक है। आँचलिक उपन्यासकार अपनी कृति में अंचल विशेष की संस्कृति का आँखों देखा चित्र प्रस्तुत करता है, उनमें यथार्थ की स्थिति महत्त्वपूर्ण और विश्वसनीय होती है। अतः वह ऐतिहासिक नहीं हो सकता। ऐतिहासिक उपन्यासों में आँचलिकता हो सकती है, जैसे वर्मा जी के उपन्यासों में, पर वे पूर्णतः आंचलिक नहीं हो सकतें ऐतिहासिक उपन्यासों में काल्पनिक तत्व भी प्रचुर मात्रा में होता है जबकि आँचलिक उपन्यासों में उसके लिए कोई स्थान नहीं है।

हिन्दी उपन्यासों में आँचलिक तत्त्व कोई नया तत्त्व नहीं हैं। 'रेणु' से बहुत पहले प्रेमचन्द, वृन्दावनलाल वर्मा, नागार्जुन आदि उपन्यासकारों के उपन्यास में और इनसे भी बहुत पहले शिवपूजन सहाय के 'देहाती दुनिया' जैसे उपन्यासों में वह विद्यमान था। उनके उपन्यास आँचलिक तत्त्व धारण करते हुए भी विशुद्ध आँचलिक उपन्यास नहीं कहे जा सकते, क्योंकि उनकी दृष्टि अधिक व्यापक है, चित्रफलक अधिक विस्तारपूर्ण है और उनकी कृतियों में वह रस नहीं जिसे 'आँचलिक रस' कह सकें। प्रेमचन्द के 'प्रेमाश्रम', 'कर्मभूमि, 'रंगभूमि' और 'गोदान' में बनारस 
Himalayan J. Soc. Sci. \& Humanities ISSN: 0975-9891

Vol. 14, (2019) 33-39

और उसके समीपवर्ती ग्रामीण अंचल के सजीव चित्र मिलते हैं, उनके पात्र अपनी धरती के पुत्र हैं, उनकी वेश-भूषा ग्रामीण तो है ही, अंचल विशेष की परिचायक भी है। वृन्दावनलाल वर्मा के ऐतिहासिक उपन्यासों में भी आँचलिक तत्त्व तो हैं पर उन्हें विशुद्ध आँचलिक उपन्यास नहीं मान सकते।

यद्यपि 'आँचलिक उपन्यास' शब्द हिन्दी में 'रेणु' में 'मैला आँचल' के प्रकाशित होने पर ही प्रयोग में आया पर उससे पूर्व भी आँचलिक उपन्यास थे, इसे अस्वीकार नहीं किया जा सकता। उनमें त्रुटियाँ हो सकती हैं, पर आँचलिक उपन्यास का मुख्य तत्त्व अंचल-विशेष के जन-जीवन का समग्र चित्रण है। वे आँचलिक नहीं कहे गये, उनमें कदाचित् आँचलिकता पर आग्रह भी उतना नहीं था जितना 'रेणु' के उपन्यासों में, तथापि उनकी आँचलिकता असंदिग्ध है। इन उपन्यासों को हम दो वर्गों में बाँट सकते हैं- नागरिक जीवन से सम्बद्ध तथा ग्रामीण जीवन से सम्बद्ध ।

हिन्दी में आँचलिक उपन्सास लिखने का श्रेय वस्तुतः बिहार के उपन्यासकारों को है। बिहार से इस क्षेत्र में सर्वप्रथम पदार्पण करने वाले थे नागार्जुन। 'बलचनमा' 3 तथा 'वरुण के बेटे'4 में आँचलिक तत्त्व प्रधान हैं। चरित्र-चित्रण, कथा की अन्विति, कथानक का आँचलिक आधार, वातावरण की सजीवता, जन-जीवन तथा संस्कृति का विशद चित्रण, भाषा सभी दृष्टि से 'बलचनमा' में सफल आँचलिक उपन्यास के गुण विद्यमान हैं। बलचनमा का चरित्र आँचलिक वातावरण की देन है, उसके व्यक्तित्त्व का निर्माण अंचल का योगदान है। लोक-गीतों का प्रयोग भी 'बलचनमा' में मिलता है-

सन्ति हे मजरल आमक बाग

कुहू कुहू चिकररा कोईलिया

झींगुर गावण फाग

कंत हमर परदेश बसइ छथि

बिसरि राम अनुराग

इन गीतों की भाषा ही नहीं पात्रों की भाषा भी जनभाषा है, बिहार के ग्रामीण क्षेत्रों में बोली जाने वाली भाषा है। 'बलचनमा' में भौगोलिक रिथति के चित्रण के अतिरिक्त अन्य आँचलिक तत्त्व पूरी सफलता के साथ प्रस्तुत किए गये हैं, अतः उसमें आँचलिक रस का स्रोत अविरल वेग से प्रवाहित होता है।

नागार्जुन का दूसरा आँचलिक उपन्यास है- 'वरुण के बेटे' जिसमें मलाही-गोंढ़ियार ग्राम के अंचल से सम्बद्ध मछुओं की आर्थिक, सामाजिक और राजनीतिक स्थिति का सजीव चित्र प्रस्तुत किया गया है। स्त्रियों के गीतों, लोकभाषा, अंचल की भौगोलिक स्थिति के चित्रण आदि ने इसे और भी सफल आंचलिक उपन्यास बना दिया है। स्त्री-पुरुषों की वेश-भूषा, मछुओं के सामाजिक सम्बन्ध, उनका जागरण, सभी 'वरुण के बेटे' को आँचलिक उपन्यास बनाते हैं। 'वरुण के बेटे' के समान ही देवेन्द्र सत्यार्थी का 'बह्मपुत्र' उपन्यास है, जिसमें ब्रह्मपुत्र नदी के तटवर्ती लोगों के जनजीवन और वहाँ की भौगोलिक स्थिति का सजीव चित्रण किया गया है। ${ }^{5}$

जाति-विशेष को लेकर जो आँचलिक उपन्यास लिखे गये हैं और जिनका संबंध ग्राम या वन-प्रदेश से है, उनमें रांगेय राघव का 'कब तक पुकारूँ'6 और देवेन्द्र सत्यार्थी का 'रथ के पहिये' उल्लेखनीय हैं। प्रथम में केवटों (नटों का एक विभेद) के जीवन का, रीति रिवाज का- उनके दैनिक कार्यक्रम, आजीविका के साधनों नट-कौशल, स्वच्छ यौन-सम्बन्धों, शराब पीने, तम्बुओं में रहने आदि का वर्णन है। देवेन्द्र सत्यार्थी के 'रथ के पहिये' में मध्य प्रदेश के करजिया जनपद और वहाँ की गोंड जाति का जीवन चित्रण किया गया है। उनके सामाजिक रीति-रिवाज और परम्पराओं, आर्थिक जीवन के चित्रों के साथ ही साथ लेखक ने लामसेनी प्रथा, करमा-नृत्य आदि के विवरण देकर उनके साँस्कृतिक जीवन की झाँकी भी प्रस्तुत की है। परम्परा में उदयशंकर भट्ट कृत 'शेष-अशेष' भी एक आँचलिक उपन्यास है ${ }^{7}$

आँचलिक उपन्यासों के वास्तविक जन्मदाता हैं फणीश्वर नाथ रेणु, जिन्होंने बिहार के जनजीवन से सम्बन्धित दो उपन्यास 'मैला आँचल' और 'परती परिकथा' लिखकर आँचलिक उपन्यासों के क्षेत्र में क्राँति उपस्थित 
Himalayan J. Soc. Sci. \& Humanities ISSN: 0975-9891

Vol. 14, (2019) 33-39

कर दी और आलोचकों को आँचलिकता को एक पृथक् तथा स्वतंत्र विधा स्वीकार करने के लिए बाध्य हो जाना पड़ा। प्रथम उपन्यास में लेखक ने 1942 ई. से गाँधी जी के निधन तक की मेरीगंज के जन-जीवन की परिस्थतियों का जीवन्त चित्र प्रस्तुत किया है। इस गाँव के विभिन्न वर्गों का- जिन्हें उसे टोली कहा है- जीवन और उनका संघर्ष बड़ी सूक्ष्मता से धार्मिक विश्वास, रुढ़ियाँ, सामाजिक परम्पराएँ, त्योहार, पर्व, नृत्य-गीति, जीवन-स्तर, रीति-रिवाज, लोक-गीत, लोक-भाषा, लोकोक्ति, मुहावरे, राजनैतिक चेतना, आर्थिक कठिनाइयों आदि का सूक्ष्म निरीक्षण व प्रयोग करता है।

आँचलिक उपन्यास के मूल तत्त्व है- (1) भौगोलिक स्थिति का अंकन, (2) कथानक का आँचलिक आधार (3) लोक-संस्कृति का चित्रण (4) वहाँ की राजनैतिक चेतना सामाजिक, धार्मिक और आर्थिक स्थिति का चित्रण (5) जन जागरण की नई चेतना। इन मूल तत्त्वों के आधार पर ही किसी भी उपन्यास को आँचलिक उपन्यास कहा जा सकता है।

आँचलिक उपन्यासों में भौगोलिक स्थिति का अंकन अनिवार्य है। आँचलिक उपन्यास का लेखक भौगोलिक स्थिति की जानकारी करा कर पाठक के मन पर न केवल अंचल विशेष का नक्शा ही उतार देता है और उसके विश्वास पर विजय पा लेता है, अपितु यह भी बताना चाहता है कि इन्हीं भौगोलिक परस्थितियों के कारण वहाँ का जन-जीवन वैसा है जैसा उपन्यासों में चित्रित किया जाता है। जहाँ तक इन उपन्यासों के प्रकृति-चित्रण का सम्बंध है उसमें काव्यात्मकता और चित्रात्मकता अधिक होती है। वह एक ओर पाठक के मर्म को छूती है, और दूसरी पाठक वहाँ की मिट्टी की सोंधी महक और ताज़गी का अनुभव करता है। बिना प्रकृति-चित्रण और भौगोलिक-स्थिति के अंकन के वह उपन्यास में अंचल का ज्वलंत चित्र प्रस्तुत कर ही नहीं सकता। वास्तविकता तो यह है कि आँचलिक उपन्यास में प्रकृति के पदार्थ ही जीते जागते पात्र होते हैं क्योंकि उनका महत्त्व पात्रों के महत्त्व से कम नहीं होता। जैसे अन्य उपन्यासों के पात्र और उनका कृतित्व एक दूसरे को प्रभावित करता है, वैसे ही नदी और उसकी बाढ़, बंजर भूमि और उसका बंजरपन, वहाँ के जन-जीवन को प्रभावित करते हैं।

आँचलिक उपन्यास का कथानक किसी जन पद विशिष्ट से सम्बद्ध होता है। 'मैला आंचल' में मैरीगंज की विभिन्न टोलियों- मालिक टोली, सिपैहिया टोली, गहलौत टोली आदि के संघर्ष का कारण मैरीगंज की भूमि है, जिसने इन विभिन्न दलों को उत्पन्न किया। उसका कथानक और पात्र भी अंचल की देन होते हैं, फिर भी वे सार्वदेशिक या सार्वभौम मानवीय तत्वों से अनुप्राणित रहते ही हैं।

आँचलिक उपन्यासों की मूलभूत विशेषताओं में सबसे महत्त्वपूर्ण तत्त्व लोक-संस्कृति का जीवन्त चित्रण है। लोक संस्कृति से हमारा तात्पर्य है वहाँ के निवासियों के रीति-रिवाज, रहन-सहन, वेशभूषा, त्यौहार-पर्व, परम्परागत मान्यताएँ, धार्मिक रुढ़ियाँ और विश्वास, उनके लोक-गीत और नृत्य, उनके मनोरंजन की विविध प्रणालियाँ, उनकी भाषा कलाएँ आदि। अंचल में होने वाले मेले-तमाशे और उनकी पृष्ठभूमि में पल्लवित विश्वासों का चित्रण आँचलिक उपन्यासकार अपने वास्तविक अनुभव के आधार पर करता है।

आँचलिक उपन्यासकार अपने उपन्यास के लिए प्रदेश विशेष को ही नहीं चुनता, एक विशिष्ट काल-खण्ड को भी चुनता है और तब प्रदेश विशेष के किसी विशिष्ट काल-खण्ड के अन्तर्गत होने वाली राजनीतिक उथल-पुथल तथा धार्मिक स्थिति का चित्रांकन करता है। भौगोलिक स्थिति और लोक संस्कृति के साथ-साथ राजनैतिक चेतना और आर्थिक स्तिति के चित्रों को समन्वित करने पर ही आँचलिकता अपने पूर्ण उत्कर्ष के साथ अवतरित होती है। राजनैतिक स्थिति के अन्तर्गत क्षेत्र विशेष की शासन-व्यवस्था, राजनैतिक चेतना, उथल-पुथल, विभिन्न विचारधाराओं के मध्य संघर्ष आदि आते हैं। उदाहरण के लिए 'मैला आँचल' में पूर्णिया के बदलते हुए (सन् 1942 के बाद से स्वतन्त्रता प्राप्ति के कुछ समय बाद तक के) राजनीतिक वातावरण के चित्र प्रस्तुत किए गए हैं, शासकों के प्रति जनता के विचार और उनके कार्यों का विवरण दिया गया है।

अंचल विशेष की राजनीतिक, सामाजिक, धार्मिक और आर्थिक स्थितियों का चित्रण करने के साथ-साथ आंचलिक उपन्यासकार उन स्थितियों के प्रति जनता की प्रतिक्रिया और नये विचारों के सम्पर्क के कारण होने वाली 
Himalayan J. Soc. Sci. \& Humanities ISSN: 0975-9891

Vol. 14, (2019) 33-39

उनकी चेतना का वर्णन भी करता है। रुढ़-अन्ध-विश्वासों, सामाजिक अनीतियों प्रशासन की ज्यादतियों, अनाचार, रिश्वत, बेईमानी, स्वार्थपरता आदि के विरुद्ध उत्क्रांति की लहर का संकेत कर वह जागरूक और सजग जनता को करवट लेती हुई समग्र चेतना को सम्पूर्ण रूप से चित्रित करता है। उदाहरण के लिए, 'मैला आँचल' में वामनदाम की मृत्यु के सम्बद्ध घटना द्वारा लेखक यह बताना चाहता है कि जनता इस तथ्य को समझने लगी है कि उसके तथा कथित रक्षक अपना कर्त्तव्य पालन नहीं कर रहे हैं, स्वार्थ-साधन में रत वे न नीति की चिंता करते हैं और न देश-हित की।

आँचलिक उपन्यासों के सम्बन्ध में प्रायः यह प्रश्न उठाया जाता है कि क्या आँचलिक उपन्यास ग्रामीण अंचल से ही सम्बद्ध होता है या वह नगर के अंचल पर भी लिखा जा सकता है? नगर के अंचल पर लिखे जाने वाले उपन्यासों को आंचलिक उपन्यास नाम न देने वालों का तर्क यह है कि नगर के कथानक से सम्बद्ध उपन्यास में प्रायः लेखक या तो सामाजिक समस्याओं में उलझ जाता है या पात्रों के मनोविश्लेषण में। अतः नगर के अंचल का जीवन्त चित्र उसमें उपस्थित नहीं हो पाता। पर यह तर्क अपने आप में लचर है। यदि अब तक लिखे गये उपन्यासों में यह त्रुटि पाई जाती है, तो यह उपन्यास विशेष का दोष है। वस्तुतः नगर के अंचल से सम्बद्ध उपन्यास में भी यदि वे ही विशेषताएँ आ जाएँ, जो ग्रामीण अंचल से सम्बद्ध आँचलिक उपन्यासों में होती हैं, तो उन्हें भी आँचलिक कहने में कोई आपत्ति नहीं होनी चाहिए। अमृतलाल का 'सेठ बांकेमल', 'बूँद और समुद्र' उदयशंकर भट्ट का 'सागर' लहरें और मनुष्य आदि ऐसे उपन्यास हैं जिनमें नगर की संस्कृति, राजनैतिक, सामाजिक अंकित किया गया है। जहाँ एक ओर लेखक ने बड़ी जाति वालों के नीच जाति की स्त्रियों से अवैध यौन-सम्बन्धों की चर्चा कर उनका नैतिक पतन दिखाया है, वहाँ दूसरी ओर राजनैतिक पतन और आर्थिक हीनता के चित्र भी अंकित किये हैं। लोक भाषा, लोक गीत और लोकोक्तियों के प्रयोग से स्थानीय रंग और भी भास्वर हो उठे हैं।

स्थानीय लोक-भाषा का प्रयोग लेखक ने दो प्रकार से किया है- वातावरण के यथातथ्य चित्रण में और पात्रों के संवाद में। इससे स्वाभाविकता तो आ गयी है, पर शब्दों के अर्थ फुटनोट में देने पर भी दुरूहता और बोझिलता पूरी तरह जा नहीं पायी है। प्रकृति चित्रण और भौगोलिक स्थिति के निर्देश ने उसकी आँचलिकता को ओर भी गहरा कर दिया है। आँचलिक उपन्यास का एक तत्व यह भी माना जा सकता है कि वह जन-जागरण का सन्देश दे। इस दृष्टि से भी 'मैला-आँचल' सफल उपन्यास कहा जायेगा। तस्कर-व्यापार, जमींदार-तहसीलदार के अत्याचार, अनैतिक सम्बन्ध, राज-नीतिक नेताओं की धांधली, वर्ग-संघर्ष आदि के चित्रों द्वारा लेखक ने अप्रत्यक्ष रूप से इन सब बुराइयों से बचने का संदेश दिया है। इन सब विशेषताओं एवं तात्विकता ने ही आँचलिकता को नव्य विधात्मक स्वीकृति दिलवायी।

रेण का 'परती परिकथा' उनका दूसरा आँचलिक उपन्यास है जिसमें पूर्णिया जिले के 'परानपुरा' गाँव और उसके आस-पास के अंचल का सजीव चित्र प्रस्तुत किया गया है। इस उपन्यास में ग्रामीण प्रेम-व्यापारों के प्रसंगों द्वारा वहाँ का सामाजिक जीवन मूर्तिमान किया गया है। जहाँ तक लोक संस्कृति के चित्रण की बात है, वह इस उपन्यास में 'मैला आँचल' से भी अधिक महत्त्वपूर्ण बना दी गई है। लोक कथाएँ- कोसी मैया की कथा, लोक-गीत कथा जैसे सुन्दरी नैका की कथा, लोक गीत जैसे-

हा, रे पन कउवा,

सावन-भादव केर उमड़ल नदिया

भांसि डोल मैया केर बेड़वा रे, पन कउवा।।

लोक-प्रथाएँ- शामा चकेवा का खेल तथा लोकभाषा, अंग्रेजी के अपभ्रंश शब्दों का प्रयोग जनपद की संस्कृति को साकार कर देते हैं, परन्तु कहीं-कहीं यह सब खलने लगता है। उदाहरण के लिए जब लेखक चिड़ियाओं की निरर्थक ध्वनियों को भी ज्यों का त्यों उतार देने का प्रयास करता है- 'तुर तुत्तू उ उ, तु उ, तू', तो 
Himalayan J. Soc. Sci. \& Humanities ISSN: 0975-9891

Vol. 14, (2019) 33-39

पाठक ऊबने लगता हे, कहीं-कहीं झुँझलाने भी लगता है। पर कहीं-कहीं यह अत्यधिक स्वाभाविक भी लगता है और वातावरण को अतीव सजीव बना देता है।

ग्रामीण अंचलों से सम्बद्ध अन्य उल्लेखनीय उपन्यास है- रामदरश मिश्र का 'पानी के प्राचीर'8 और शैलेश मटियानी का 'होल्दार'9। पहले में गोरखपुर जिले के राप्ती और गोरी नदियों से घिरे भूभाग के कल्पित गाँव पाँडेपुरवा की कथा है, तो दूसरे में कुमायूँ के पर्वतीय अंचल की झाँकी प्रस्तुत की गई है।

रांगेय राघव का 'काका', उदयशंकर भट्ट का 'सागर, लहरें और मनुष्य', और शिवप्रसाद मिश्र 'रुद्र' का 'बहती गंगा' नागरिक अंचल से संबद्ध हैं। 10 'काका' मथुरा के जन-जीवन का सामाजिक चित्र है। उसके पात्रों का चरित्र-विकास अंचल के कारण ही होता है। वातावरण तथा प्रकृति के चित्र (यमुना तट आदि के) स्थानीय भाषा का प्रयोग- 'आज लाला कहीं चोट खाकर आये हो, तभी यहाँ इतनी उसके दिखा रहे हो' अथवा 'अरे तेरे मुँह पै आग बराऊँ' आदि भी उसे आँचलिक बनाने में सहायक होते हैं। अतः नागरीय अंचल से सम्बद्ध प्रथम उपन्यास होने के कारण उसका अपना महत्त्व है।

'सागर, लहरें और मनुष्य' में लेखक ने बम्बई के उपनगर 'बारसोवा' के मछुओं और 'माहीम' के कोलियों का जीवन चित्रित किया है। इन दोनों जातियों का रहन-सहन, स्वभाव, रीति-रिवाज, त्योहार जैसे नारियल-पूर्णिमा, यौन-सम्बन्ध, आर्थिक स्थिति- गरीबी, सिनेमा का प्रभाव आदि इस सजीवता के साथ चित्रित किए गए हैं कि ऐसा लगता है कि हम उपन्यास न पढ़कर बम्बई के समुद्र तट पर खड़े मछुओं को देख रहे हैं। उनके गीत और नृत्य उनके सांस्कृतिक जीवन की झाँकी प्रस्तुत करते हैं, तो शराब, गांजा आदि मादक द्रव्यों का प्रयोग, उनके सेवन के बाद लड़ाई-झगड़े, मार-पीट, गाली-गलौज उनकी हीन दशा का संकेत करते हैं। इस तरह उपन्यास में मछुओं का जीवन पूरी तरह उतर आया है।

रुद्र का 'बहती गंगा' ऐतिहासिक पृष्ठभूमि पर लिखा गया आंचलिक उपन्यास है जिसमें काशी की लगभग 200 वर्ष की (1750-1950) सामाजिक सांस्कृतिक एवं राजनीतिक चेतना का चित्र प्रस्तुत किया गया है। इसमें काशी की समग्र समाज- राजा-रंक, व्यापारी, मजदूर, पण्डे-पुरोहित आदि की विकासोन्मुख चेतना को वाणी दी गई है। काशी की मस्ती, उसका पौरुष, उसके सामाजिक मूल्य, धार्मिक विश्वास, उसकी दुर्बलताएँ, अलौकिक गुण, आन-बान, व्यवसाय, रूप-विन्यास, आचार-विचार आदि इस उपन्यास में साकार हो उठे हैं। इनकी भाषा में तनिक भी मिलावट नहीं है, वह सीधी मुहावरेदार, सरस सूक्तियों और लहरियादार शब्दावली से भरी तथा भावनानुकूल है।

इनके अतिरिक्त डॉ. शरण बिहारी गोस्वामी के 'पूछरी का लोठा'11 ब्रज भाषा का प्रथम उपन्यास होने के साथ-साथ आँचलिक महत्त्व भी रखता है। आँचलिकता के सभी तत्त्वों का सुघड़ उन्मेष इसमें स्पष्ट देखा जा सकता है। राजाराम शास्त्री का हरियाणवी भाषा में विरचित 'झाडू फिरी' 12 हरियाणवी का पहला उपन्यास तो है ही सही, समग्रतः आँचलिक भी है। अन्य आँचलिक उपन्यासों में राजेश शर्मा का 'कौए' उपन्यास ${ }^{13}$ भी महत्त्वपूर्ण है।

स्थानीय भाषा का प्रयोग आँचलिक उपन्यासों की विशेषता है। वातावरण के यथार्थ चित्रण तथा स्थानीय रंग लाने के लिए स्थानीय भाषा का प्रयोग किया जाता है, पर उसका आधिक्य कई समस्याएँ उपस्थित करता है वस्तुतः लोक-भाषा का प्रयोग तीन उद्देश्यों से किया जाता है- (क) स्थानीय प्रभाव उत्पन्न करने के लिए (ख) यथार्थवाद का कलात्मक पुट देने के लिए तथा (ग) हास्य-विनोद और व्यंग्य की सृष्टि के लिए। यदि लोकभाषा का प्रयोग इन तीनों उद्देश्यों की पूर्ति करता है, तो वह श्लाघनीय है पर यदि वह दुरुहता उत्पन्न करता है, पाठक के मन में ऊब उत्पन्न करता है, तो निश्चय ही त्याज्य है। आँचलिक उपन्यासकार को सतर्क रहना चाहिए कि उपन्यास स्थानीय बोलियों का व्यवस्थाहीन और अराजकतापूर्ण जमघट बनकर न रह जाय।

उपन्यासों में आँचलिकता मानवीय संवेदना को उभारती है। जन-जीवन को जिस व्यापकता के साथ आँचलिक उपन्यास चित्रित करता है, उतनी व्यापकता अन्य उपन्यासों में नहीं देखी जाती। वह हमें अंचल विशेष की संस्कृति, धार्मिक विश्वास, प्रथाओं, रहन-सहन, रीति-रिवाज, भौगोलिक सीमाओं से परिचित करता है। वर्तमान राजनीतिक तथा आर्थिक समस्याओं का चित्रण करता है। भारत की विभिन्न आँचलिक संस्कृतियों के भेद में अभेद 
दिखाकर सांस्कृतिक एकीकरण और भावात्मक एकता का स्तुत्य प्रयास करता है। कतिपय आलोचकों का मत है कि आँचलिक उपन्यास कुछ विशेष रूचि के पाठकों को ही प्रमुदित कर सकते हैं, पर यह ठीक नहीं। अब तक जो देश-विदेश में आँचलिक उपन्यास लिखे गये हैं, उन्हें देखकर यह नहीं कहा जा सकता। हार्डी के वैसेक्स नाविल मार्क, ट्वेन का 'लाइफ आन मिसीसिपी' फणीश्वर रेणु का 'मैला आँचल' इसके प्रमाण हैं। अतः आँचलिक उपन्यास ने न केवल साहित्यकार के लिए नए क्षितिज खोले हैं, अपितु उसकी शक्ति भी असीम है।

\section{संदर्भ ग्रंथ}

1. रेणु, फणीश्वरनाथ, मैला आंचल, परती परिकथा

2. सहाय, शिवपूजन, देहाती दुनियाँ

3. नागार्जुन, बलचनमा

4. नागार्जुन, वरुण के बेटे

5. सत्यार्थी देवेन्द्र, ब्रह्मपुत्र, रथ के पहिये

6. राघव, रांगेय, कब तक पुकारूँ, काका

7. भट्ट, उदयशंकर, शेष-अशेष, सागर, लहरें और मनुष्य

8. मिश्र, रामदरश, पानी के प्राचीर

9. मटियानी, शैलेश, होलदार

10. मिश्र, शिव प्रसाद, 'रुद्र', बहती गंगा

11. गोस्वामी, डॉ. शरण बिहारी, पूछरी का लोग

12. शास्त्री, राजाराम, झाडूफिरी

13. शर्मा, राजेश, कौए 